\title{
RANK OF LINEAR AND QUADRATIC COMBINATIONS OF MATRICES*
}

\author{
CH.R. JOHNSON ${ }^{\dagger}$, J.M. PEÑA ${ }^{\ddagger}$, AND T. SZULC $\S$
}

\begin{abstract}
In this paper, the rank of some combinations of matrices is analysed. In particular, the rank of all matrices on the line joining two rank 1 matrices is characterized, and the rank of convex combinations of two matrices and quadratic combinations of three matrices is studied. Presented results concern the problem of robustness of rank under certain kinds of perturbations of a matrix.
\end{abstract}

Key words. Rank, Full rank factorization, Linear combinations of matrices, Convex combinations, Quadratic combinations, P-matrices.

AMS subject classifications. 15A15, $15 \mathrm{~A} 45$.

1. Introduction. We are generally interested in when two rank $k$ matrices $A$ and $B$ exhibit rank $k$ among all matrices on the line segment joining them. In the case $k=1$, we give a complete characterization (and more), and in the case $k>1$ (and not full rank matrices), we give a partial characterization. Then we consider quadratic combinations of three matrices of the same size and full rank. It is characterized when all combinations have full rank. These results may be compared to the classic fact in the square case (e.g. $[4,5])$ that all combinations of two matrices are full rank, unless $A^{-1} B$ has a negative real eigenvalue.

Let us introduce some basic notations. Given a field $\mathbb{F}$ (with either $\mathbb{F}=\mathbb{R}$ or $\mathbb{F}=\mathbb{C}$ ) and two matrices $A, B$ with entries in $\mathbb{F}$, let

$$
\mathcal{S}_{u}(A, B)=\{t A+u B ; t, u \in \mathbb{F}, \text { not both } 0\}
$$

be the subspace generated by $A$ and $B$, except for the 0 matrix;

$$
\mathcal{L}(A, B)=\{t A+(1-t) B ; t \in \mathbb{F}\},
$$

the affine line generated by $A$ and $B$; and

$$
\mathcal{L} S(A, B)=\{t A+(1-t) B ; t \in[0,1]\},
$$

the line segment joining $A$ and $B$, that is, the set of convex combinations of $A$ and $B$.

Finally, denote the spectral norm of a square matrix $A$ by $\|A\|_{2}$. Recall that the spectral norm coincides with the maximal singular value of the matrix.

The paper is organized as follows. Section 2 analyzes the case of linear combinations of rank 1 matrices. Section 3 presents a result on the rank of convex combinations of two matrices. Section 4 presents results

\footnotetext{
*Received by the editors on July 5, 2019. Accepted for publication on February 2, 2020. Handling Editor: Michael Tsatsomeros. Corresponding Author: Juan Manuel Peña.

†Department of Mathematics, College of William and Mary, Williamsburg, VA 23185, USA (crjohn@wm.edu).

‡Departamento de Matemática Aplicada. Universidad de Zaragoza, 50009 Zaragoza, Spain (jmpena@unizar.es). Supported by the Spanish Research grant PGC2018-096321-B-I00 (MCIU/AEI), by Gobierno de Aragón (E41-17R), and by Feder 20142020 "Construyendo Europa desde Aragón".

$\S$ Faculty of Mathematics and Computer Science, Adam Mickiewicz University, Umultowska 87, 61-614 Poznan, Poland (tszulc@amu.edu.pl).
} 
on quadratic combinations of three matrices. Finally, Section 5 presents some results on full rank matrices related to P-matrices.

2. Linear combinations of two rank 1 matrices. The following result characterizes the rank of all matrices on the subspace generated by two rank 1 matrices $A$ and $B$ (that is, of matrices in $\mathcal{L}(A, B)$ ).

Theorem 2.1. Suppose that $A, B \in \mathbb{F}^{m, n}$ are rank 1 matrices: $A=a \alpha^{T}, B=b \beta^{T}, a, b \in \mathbb{F}^{m}, \alpha, \beta \in \mathbb{F}^{n}$, $a, b, \alpha, \beta \neq 0$. Then:

a) if $b$ is a multiple of $a(b=r a, r \neq 0)$, or $\beta$ is a multiple of $\alpha(\beta=s \alpha, s \neq 0)$, but not both, then all matrices in $\mathcal{S}_{u}(A, B)$ are rank 1 ;

b) if $b$ is a multiple of a and $\beta$ is a multiple of $\alpha$, then all matrices in $\mathcal{S}_{u}(A, B)$ are rank 1 , except ones of the form $t A+u B$, with $t=-$ urs, that are 0 ;

c) if neither $b$ is a multiple of $a$, nor $\beta$ is a multiple of $\alpha$, then all matrices in $\mathcal{L}(A, B)$ are rank 2 , except for $A$ and $B$.

Proof. For part a), $C \in \mathcal{S}_{u}(A, B)$ satisfies $C=t A+u B$. Without loss of generality, assume $b=r a$, but $\beta \neq s \alpha$. Then, $C=\operatorname{ta} \alpha^{T}+u r a \beta^{T}=a(t \alpha+u r \beta)^{T}$. Since $a \neq 0$ and $t \alpha+u r \beta \neq 0$ (else $\beta$ would be a multiple of $\alpha$ ), $C$ is rank 1 .

For part b), $C \in \mathcal{S}_{u}(A, B)$ is of the form $t a \alpha^{T}+u r s a \alpha^{T}$, which is a nonzero multiple of $a \alpha^{T}$ (and, thus, rank 1), unless $t=-$ urs, in which case $C=0$.

For part c), $C \in \mathcal{L}(A, B)$, note that rank $C$ can be at most 2 , so that if $\operatorname{rank} C \geq 2$, it is 2 . If any 2 -by-2 submatrix has rank 2 , then $C$ has rank 2 . There must be corresponding submatrices meeting the hypothesis, else the entire pair would fail. Either there is such a submatrix pair with a common nonzero entry in each matrix, or not. Suppose there is. After normalization (independently of each, which may be absorbed in the coefficients of a linear combination), we have

$$
t\left(\begin{array}{c}
1 \\
w
\end{array}\right)\left(\begin{array}{ll}
1 & x
\end{array}\right)+(1-t)\left(\begin{array}{l}
1 \\
y
\end{array}\right)\left(\begin{array}{ll}
1 & z
\end{array}\right)
$$

whose determinant, after algebra, is

$$
t(1-t)((w-y)(x-z))
$$

Since neither $t$ nor $1-t=0$ ( else we would have one of $A$ or $B$ ) and $w \neq y, x \neq z$, this cannot be 0 . Thus, the determinant is nonzero and the rank is 2 , completing the proof. If no nonzero matches occur, cases may be considered to the same end (also special form, etc.)

We shall close this section with some concrete examples illustrating Theorem 2.1. Let $A=a \alpha^{T}, B=b \beta^{T}$, with $a=(1,2)^{T}, \alpha=(1,1,0)^{T}, b=(2,4)^{T}$ and $\beta=(0,1,2)^{T}$. Then it is easy to see that for all $t, u \in t, u \in \mathbb{F}$ with $|t|^{2}+|u|^{2}>0$ we have

$$
\operatorname{rank}(t A+u B)=\operatorname{rank}\left(\begin{array}{ccc}
t & t+2 u & 4 u \\
2 t & 2 t+4 u & 8 u
\end{array}\right)=\operatorname{rank}\left(\begin{array}{ccc}
t & t+2 u & 4 u \\
0 & 0 & 0
\end{array}\right)=1 .
$$

Now let $A=a \alpha^{T}, B=b \beta^{T}$, with $a=(1,1,0)^{T}, \alpha=(-2,3)^{T}, b=(2,2,0)^{T}$ and $\beta=(2,-3)^{T}$. Then it is easy to see that for all $t, u \in \mathbb{F}$ with $t \neq 2 u$ we have

$$
\operatorname{rank}(t A+u B)=\operatorname{rank}\left(\begin{array}{cc}
-2 t+4 u & 3 t-6 u \\
-2 t+4 u & 3 t-6 u \\
0 & 0
\end{array}\right)=\operatorname{rank}\left(\begin{array}{cc}
-2 t+4 u & 3 t-6 u \\
0 & 0 \\
0 & 0
\end{array}\right)=1 .
$$


In the case $t=2 u$, then

$$
\operatorname{rank}(t A+u B)=\operatorname{rank}\left(\begin{array}{cc}
0 & 0 \\
0 & 0 \\
0 & 0
\end{array}\right)=0
$$

Finally, let $A=a \alpha^{T}, B=b \beta^{T}$, with $a=(-1,2)^{T}, \alpha=(1,0,1)^{T}, b=(1,3)^{T}$ and $\beta=(0,1,1)^{T}$. Then it is easy to see that for any $t \in \mathbb{F} \backslash\{0,1\}$ we have

$$
\operatorname{rank}(t A+(1-t) B)=2
$$

3. Rank of convex combinations of two matrices. We now present a result on the rank of convex combinations of two matrices $A, B$ (that is, of matrices in $\mathcal{L} S(A, B)$ ).

Theorem 3.1. Let $A, B \in \mathbb{C}^{m \times n}, m \geq n$, and let $\operatorname{rank}(A)=\operatorname{rank}(B)=k, 1 \leq k \leq n$. Moreover, assume that there is a common factor $C_{A, B}$ in the full rank factorization of $A$ and $B$, and let

$$
\operatorname{Spec}\left(M_{1}^{-1} M_{2}\right) \cap(-\infty, 0]=\emptyset
$$

if $A=C_{A, B} S_{A}$ and $B=C_{A, B} S_{B}$ with $C_{A, B} \in \mathbb{C}^{m \times k}$,

$$
M_{1}=\left(\begin{array}{cc}
S_{A} S_{A}^{*} & 0 \\
-I & I
\end{array}\right), \quad M_{2}=\left(\begin{array}{cc}
S_{B} S_{B}^{*} & S_{A} S_{B}^{*}+S_{B} S_{A}^{*}-S_{A} S_{A}^{*}-S_{B} S_{B}^{*} \\
0 & I
\end{array}\right)
$$

or

$$
\operatorname{Spec}\left(P_{1}^{-1} P_{2}\right) \cap(-\infty, 0]=\emptyset
$$

if $A=S_{A} C_{A, B}$ and $B=S_{B} C_{A, B}$ with $C_{A, B} \in \mathbb{C}^{k \times n}$,

$$
P_{1}=\left(\begin{array}{cc}
S_{A}^{*} S_{A} & 0 \\
-I & I
\end{array}\right), \quad P_{2}=\left(\begin{array}{cc}
S_{B}^{*} S_{B} & S_{A}^{*} S_{B}+S_{B}^{*} S_{A}-S_{A}^{*} S_{A}-S_{B}^{*} S_{B} \\
0 & I
\end{array}\right) .
$$

Then all convex combinations of $A$ and $B$ have rank $k$.

Proof. We restrict ourselves to the case when (3.1) holds (the proof in the case when (3.2) holds is similar).

It is well-known that for any matrix $X$ we have

$$
\operatorname{rank}(X)=\operatorname{rank}\left(X^{*} X\right)=\operatorname{rank}\left(X X^{*}\right)
$$

Hence, for any $\alpha \in[0,1]$, we get

$$
\begin{aligned}
\operatorname{rank}(\alpha A+(1-\alpha) B) & =\operatorname{rank}\left(\alpha C_{A, B} S_{A}+(1-\alpha) C_{A, B} S_{B}\right) \\
& =\operatorname{rank}\left(C_{A, B}\left(\alpha S_{A}+(1-\alpha) S_{B}\right)\right)= \\
& =\operatorname{rank}\left(\left(C_{A, B}\left(\alpha S_{A}+(1-\alpha) S_{B}\right)\right)^{*}\left(C_{A, B}\left(\alpha S_{A}+(1-\alpha) S_{B}\right)\right)\right) \\
& \left.=\operatorname{rank}\left(\left(\alpha S_{A}+(1-\alpha) S_{B}\right)^{*} C_{A, B}^{*} C_{A, B}\left(\alpha S_{A}+(1-\alpha) S_{B}\right)\right)\right) .
\end{aligned}
$$

Observe that $C_{A, B}^{*} C_{A, B} \in \mathbb{C}^{k \times k}$ is a positive definite matrix, and therefore, it admits a Cholesky factorization, i.e.,

$$
C_{A, B}^{*} C_{A, B}=L L^{*}
$$


where $L$ is a nonsingular lower triangular $k \times k$ matrix. So, by (3.5) and (3.3), (3.4) becomes

$$
\operatorname{rank}(\alpha A+(1-\alpha) B)=\operatorname{rank}\left(L^{*}\left(\alpha S_{A}+(1-\alpha) S_{B}\right)\right),
$$

which, as $L^{*}$ is nonsingular, yields

$$
\operatorname{rank}(\alpha A+(1-\alpha) B)=\operatorname{rank}\left(\alpha S_{A}+(1-\alpha) S_{B}\right)
$$

for any $\alpha \in[0,1]$. To complete the proof, we recall that $S_{A}, S_{B} \in \mathbb{C}^{k \times n}$ have full row rank. So, keeping in mind (3.1), by Theorem 2 from [5], we get that all convex combinations of $S_{A}, S_{B}$ have full row rank $k$. Thus, by (3.6), for any $\alpha \in[0,1]$, we obtain

$$
\operatorname{rank}(\alpha A+(1-\alpha) B)=\operatorname{rank}\left(\alpha S_{A}+(1-\alpha) S_{B}\right)=k,
$$

which completes the proof.

4. Quadratic combinations of three matrices. We now present results on quadratic combinations of three matrices.

Theorem 4.1. Let $A, B, C \in \mathbb{C}^{m \times n}$, let $\operatorname{rank}(A+B+C)=\operatorname{rank}(C)=m$ and let

$$
P=\left(\begin{array}{cc}
A+B+C & 0 \\
I_{n} & I_{n}
\end{array}\right) \text { and } Q=\left(\begin{array}{cc}
C & A \\
0 & I_{n}
\end{array}\right)
$$

$A$ necessary and sufficient condition for all quadratic combinations of $A, B, C$ (i.e., for all matrices $\alpha^{2} A+$ $\alpha B+C, \alpha \in[0,1])$ to be of rank $m$ is that

$$
\operatorname{Spec}\left(H_{1} H_{2}^{-1}\right) \cap(-\infty, 0]=\emptyset,
$$

where

$$
H_{1}=\left(\begin{array}{cc}
P P^{*} & 0 \\
-I_{n+m} & I_{n+m}
\end{array}\right), \quad H_{2}=\left(\begin{array}{cc}
Q Q^{*} & P Q^{*}+S_{B} S_{A} Q P^{*}-P P^{*}-Q Q^{*} \\
0 & I_{n+m}
\end{array}\right) .
$$

Proof. First observe that $\alpha^{2} A+\alpha B+C$ is a Schur complement of $I_{n}$ in

$$
R_{\alpha}=\left(\begin{array}{cc}
\alpha(A+B)+C & (1-\alpha) A \\
\alpha I_{n} & I_{n}
\end{array}\right) .
$$

Then, by a well known property of a rank of a block matrix, we have

$$
\operatorname{rank}\left(R_{\alpha}\right)=\operatorname{rank}\left(\alpha^{2} A+\alpha B+C\right)+\operatorname{rank}\left(I_{n}\right),
$$

and therefore, the full rank property of $\alpha^{2} A+\alpha B+C$ is equivalent to the full rank property of $R_{\alpha}$ for all $\alpha \in[0,1]$. Now it is easy to see that for $R_{\alpha}$ we have

$$
\left(\begin{array}{cc}
\alpha(A+B)+C & (1-\alpha) A \\
\alpha I_{n} & I_{n}
\end{array}\right)=\alpha\left(\begin{array}{cc}
A+B+C & 0 \\
I_{n} & I_{n}
\end{array}\right)+(1-\alpha)\left(\begin{array}{cc}
C & A \\
0 & I_{n}
\end{array}\right),
$$

which by (4.7) becomes

$$
\left(\begin{array}{cc}
\alpha(A+B)+C & (1-\alpha) A \\
\alpha I_{n} & I_{n}
\end{array}\right)=\alpha P+(1-\alpha) Q .
$$

Then the assertion follows from Theorem 2 of [6]. 
REMARK 4.2. In Theorem 4.1, one can assume that $\operatorname{rank}(A+B+C)=\operatorname{rank}(C)=n$ and the condition (4.8) is changed accordingly.

Theorem 4.3. Let $A, B, C \in \mathbb{C}^{n \times n}$ and let $A+B+C$ and $C$ be nonsingular. $A$ necessary and sufficient condition for all quadratic combinations of $A, B, C$ to be nonsingular is that

$$
\operatorname{Spec}\left(\left(\begin{array}{cc}
A+B+C & 0 \\
I_{n} & I_{n}
\end{array}\right)\left(\begin{array}{cc}
C & A \\
0 & I_{n}
\end{array}\right)^{-1}\right) \cap(-\infty, 0]=\emptyset .
$$

Proof. By the arguments used in the proof of Theorem 4.1, we have that nonsingularity of $\alpha^{2} A+\alpha B+C$ for all $\alpha \in[0,1]$ is equivalent to the nonsingularity of all convex combinations of

$$
\left(\begin{array}{cc}
A+B+C & 0 \\
I_{n} & I_{n}
\end{array}\right) \text { and }\left(\begin{array}{cc}
C & A \\
0 & I_{n}
\end{array}\right)
$$

Then the assertion follows by [5].

REMARK 4.4. Another characterization of nonsingularity of quadratic combinations of three matrices can be found in [9].

From now on, $X^{+}$will denote the Moore-Penrose generalized inverse of a matrix $X$.

Theorem 4.5. Let $A, B, C \in \mathbb{C}^{m \times n}$, let $\operatorname{rank}(A+B+C)=\operatorname{rank}(C)=m$ and let

$$
\operatorname{Spec}\left(\left(\begin{array}{cc}
A+B+C & 0 \\
I_{n} & I_{n}
\end{array}\right)\left(\begin{array}{cc}
C & A \\
0 & I_{n}
\end{array}\right)^{+}\right) \cap(-\infty, 0]=\emptyset .
$$

Then all quadratic combinations of $A, B, C$ are of rank $m$.

Proof. Using the notation of our Theorem 4.1, we start by observing that for the $(m+n) \times 2 n$ matrix $Q$ of (4.7) we have

$$
\operatorname{rank}(Q)=\operatorname{rank}(C)+\operatorname{rank}\left(I_{n}\right)=m+n
$$

So, $Q$ is a full (row) rank matrix, and therefore, $Q Q^{*}$ is a nonsingular $(m+n) \times(m+n)$ matrix. Next, by (4.11), the $(m+n) \times(m+n)$ matrix

$$
\left(\begin{array}{cc}
A+B+C & 0 \\
I_{n} & I_{n}
\end{array}\right)\left(\begin{array}{cc}
C & A \\
0 & I_{n}
\end{array}\right)^{+}=\left(\begin{array}{cc}
A+B+C & 0 \\
I_{n} & I_{n}
\end{array}\right) Q^{+}
$$

is nonsingular and, from Theorem 2 of [5], we get that all convex combinations of the matrix (4.13) and $I_{m+n}$ are nonsingular, i.e., for all $\alpha \in[0,1]$ we have

$$
\operatorname{rank}\left(\alpha\left(\begin{array}{cc}
A+B+C & 0 \\
I_{n} & I_{n}
\end{array}\right)\left(\begin{array}{cc}
C & A \\
0 & I_{n}
\end{array}\right)^{+}+(1-\alpha) I_{m+n}\right)=m+n
$$

It is well-known that for a full row rank matrix $Q$, taking into account that $Q Q^{*}$ is nonsingular, we have $Q^{+}=Q^{*}\left(Q Q^{*}\right)^{-1}$ and $Q Q^{+}=I_{m+n}$. Then from (4.14) we obtain

$$
m+n=\operatorname{rank}\left(\left(\alpha\left(\begin{array}{cc}
A+B+C & 0 \\
I_{n} & I_{n}
\end{array}\right)+(1-\alpha)\left(\begin{array}{cc}
C & A \\
0 & I_{n}
\end{array}\right)\right) Q^{+}\right)
$$


which, as $\operatorname{rank}\left(Q^{+}\right)=m+n$ by (4.12), from a property of the product of matrices becomes

$$
\begin{aligned}
m+n & =\operatorname{rank}\left(\alpha\left(\begin{array}{cc}
A+B+C & 0 \\
I_{n} & I_{n}
\end{array}\right)+(1-\alpha)\left(\begin{array}{cc}
C & A \\
0 & I_{n}
\end{array}\right)^{+}\right) \\
& =\operatorname{rank}\left(\begin{array}{cc}
\alpha(A+B)+C & (1-\alpha) A \\
\alpha I_{n} & I_{n}
\end{array}\right) .
\end{aligned}
$$

To complete the proof, we use arguments from the proof of Theorem 4.1. So, using (4.9) and (4.10), (4.15) becomes

$$
m+n=\operatorname{rank}\left(\alpha^{2} A+\alpha B+C\right)+n
$$

So, from the last equality we get $m=\operatorname{rank}\left(\alpha^{2} A+\alpha B+C\right)$, which completes the proof.

5. P-matrices and some results on full rank matrices. Let us recall that a principal submatrix of an $n$-by- $n$ matrix $A$ is a submatrix using the same rows and columns of $A$, and its determinant will be the corresponding principal minor. The matrix $A$ is called a $\mathrm{P}$-matrix if all its principal minors are positive. $\mathcal{D}_{[0,1]}^{p}$ will denote the set of all diagonal $p$-by- $p$ matrices with diagonal entries on the interval $[0,1]$. In our next results, the order of the identity matrices will follow from the context.

Theorem 5.1. Let $A, B, C \in \mathbb{R}^{m \times n}$ and let $\operatorname{rank}(A+B+C)=\operatorname{rank}(B+C)=\operatorname{rank}(C)=m$. If the $2 m \times 2 m$ matrix

$$
\left(\begin{array}{cc}
A C^{+}+B C^{+}+I & -I \\
-A C^{+} & I
\end{array}\right)
$$

is a $\mathrm{P}$-matrix, then for any $\tilde{D}, D \in \mathcal{D}_{[0,1]}^{m}$, we have

$$
\operatorname{rank}(\tilde{D} D A+\tilde{D} B+C)=\operatorname{rank}(C)=m
$$

Proof. Full rank property for "extremal" matrices, i.e., for cases when diagonal matrices are identity or zero ones, follows by an assumption. By Corollary 3.2 of [2], keeping in mind the form of the matrix (5.16), for any $D \in \mathcal{D}_{[0,1]}^{m}$ the matrix

$$
D\left(A C^{+}+B C^{+}+I\right)+(I-D)\left(B C^{+}+I\right)=D A C^{+}+B C^{+}+I
$$

is a $\mathrm{P}$-matrix, and therefore, so is the matrix $\left(D A C^{+}+B C^{+}+I\right)^{-1}$. Then, by Theorem 3.3 of [5], for any $\tilde{D} \in \mathcal{D}_{[0,1]}^{m}$ the matrix

$$
\tilde{D}\left(D A C^{+}+B C^{+}+I\right)+(I-\tilde{D})
$$

is nonsingular. So, for all $\tilde{D}, D \in \mathcal{D}_{[0,1]}^{m}$, we have

$$
\begin{aligned}
m & =\operatorname{rank}\left(\tilde{D} D A C^{+}+\tilde{D} B C^{+}+\tilde{D}+I-\tilde{D}\right)=\operatorname{rank}\left(\tilde{D} D A C^{+}+\tilde{D} B C^{+}+I\right) \\
& =\operatorname{rank}\left(\tilde{D} D A C^{+}+\tilde{D} B C^{+}+C C^{+}\right)=\operatorname{rank}\left((\tilde{D} D A+\tilde{D} B+C) C^{+}\right) .
\end{aligned}
$$

Since $\operatorname{rank}\left(C^{+}\right)=m$, the assertion follows from the property of the rank of a product of matrices.

The following result is a direct consequence of Theorem 5.3. 
Electronic Journal of Linear Algebra, ISSN 1081-3810

Corollary 5.2. Let $A, B, C \in \mathbb{R}^{m \times m}$ with $A+B+C, B+C$ and $C$ nonsingular. If the $2 m \times 2 m$ matrix

$$
\left(\begin{array}{cc}
A C^{-1}+B C^{-1}+I & -I \\
-A C^{-1} & I
\end{array}\right)
$$

is a $\mathrm{P}$-matrix, then for any $\tilde{D}, D \in \mathcal{D}_{[0,1]}^{m}$ we have that the matrix $\tilde{D} D A+\tilde{D} B+C$ is nonsingular.

Theorem 5.3. Let $A, B, C \in \mathbb{R}^{m \times n}$ and let $\operatorname{rank}(A+B+C)=\operatorname{rank}(B+C)=\operatorname{rank}(C)=n$. If the $2 n \times 2 n$ matrix

$$
\left(\begin{array}{cc}
C^{+} A+C^{+} B+I & -C^{+} A \\
-I & I
\end{array}\right)
$$

is a $\mathrm{P}$-matrix, then for any $\tilde{D}, D \in \mathcal{D}_{[0,1]}^{n}$, we have

$$
\operatorname{rank}(A D \tilde{D}+B \tilde{D}+C)=\operatorname{rank}(C)=n .
$$

Proof. Full rank property for "extremal" matrices, i.e., for cases when diagonal matrices are identity or zero ones, follows by an assumption. By Corollary 3.1 of [2], keeping in mind the form of the matrix (5.18), for any $D \in \mathcal{D}_{[0,1]}^{n}$ the matrix

$$
\left(C^{+} A+C^{+} B+I\right) D+\left(B C^{+}+I\right)(I-D)=C^{+} A D+C^{+} B+I
$$

is a P-matrix. Then, by Theorem 3.4 of [5], for any $\tilde{D} \in \mathcal{D}_{[0,1]}^{m}$ the matrix

$$
\left(C^{+} A D+C^{+} B+I\right) \tilde{D}+(I-\tilde{D})
$$

is nonsingular. So, for all $\tilde{D}, D \in \mathcal{D}_{[0,1]}^{m}$, we have

$$
\begin{aligned}
m & =\operatorname{rank}\left(C^{+} A D \tilde{D}+C^{+} B \tilde{D}+\tilde{D}+I-\tilde{D}\right)=\operatorname{rank}\left(C^{+} A D \tilde{D}+C^{+} B \tilde{D}+I\right) \\
& =\operatorname{rank}\left(C^{+} A D \tilde{D}+C^{+} B \tilde{D}+C^{+} C\right)=\operatorname{rank}\left(C^{+}(A D \tilde{D}+B \tilde{D}+C)\right)
\end{aligned}
$$

Since $\operatorname{rank}\left(C^{+}\right)=m$, the assertion follows from the property of the rank of a product of matrices.

Analogously to Corollary 5.2, a similar corollary can be derived from Theorem 5.3. We shall close this section with some special cases of theorems 5.1 and 5.3.

Theorem 5.4. Let $A, B \in \mathbb{R}^{m \times n}$ and let $\operatorname{rank}(A+B)=\operatorname{rank}(2 A+B)=\operatorname{rank}(A)=$ m. If the matrix $B A^{+}$is a P-matrix, then for any $\tilde{D}, D \in \mathcal{D}_{[0,1]}^{m}$, we have

$$
\operatorname{rank}(\tilde{D} D A+\tilde{D} B+A)=m \text {. }
$$

Proof. Full rank property for "extremal" matrices, i.e., for cases when diagonal matrices are identity or zero ones, follows by an assumption. We start by the observation that, as the sum of a P-matrix and a nonnegative diagonal matrix is a P-matrix (cf. Theorem 3.1 (5) of [10]), then for any $D \in \mathcal{D}_{[0,1]}^{m}$ the matrix $B A^{+}+D+I$ is a P-matrix. Then, as also $\left(B A^{+}+D+I\right)^{-1}$ is a P-matrix, by Theorem 3.3 of [5], for any $\tilde{D} \in \mathcal{D}_{[0,1]}^{m}$ the matrix

$$
\tilde{D}\left(D+I+B A^{+}\right)+(I-\tilde{D})
$$


is nonsingular. So, for all $\tilde{D}, D \in \mathcal{D}_{[0,1]}^{m}$, we have

$$
\begin{aligned}
m & =\operatorname{rank}\left(\tilde{D}\left(D+I+B A^{+}\right)+I-\tilde{D}\right)=\operatorname{rank}\left(\tilde{D} D+\tilde{D} B A^{+}+I\right) \\
& =\operatorname{rank}\left(\tilde{D} D A+\tilde{D} B A^{+}+A A^{+}\right)=\operatorname{rank}\left((\tilde{D} D A+\tilde{D} B+A) A^{+}\right) .
\end{aligned}
$$

Since $\operatorname{rank}\left(A^{+}\right)=m$, the assertion follows from the property of the rank of a product of matrices.

Theorem 5.5. Let $A, B \in \mathbb{R}^{m \times n}$ and let $\operatorname{rank}(A+B)=\operatorname{rank}(2 A+B)=\operatorname{rank}(A)=n$. If $A^{+} B$ is a P-matrix, then for any $\tilde{D}, D \in \mathcal{D}_{[0,1]}^{n}$, we have

$$
\operatorname{rank}(A D \tilde{D}+B \tilde{D}+A)=n
$$

Proof. Full rank property for "extremal" matrices, i.e., for cases when diagonal matrices are identity or zero ones, follows by an assumption. By the argument used in the proof of Theorem 5.4 , for any $D \in \mathcal{D}_{[0,1]}^{m}$ the matrix $A^{+} B+D+I$ is a P-matrix. Then, by Theorem 3.4 of [5], for any $\tilde{D} \in \mathcal{D}_{[0,1]}^{m}$ the matrix

$$
\left(D+I+B A^{+}\right) \tilde{D}+(I-\tilde{D})
$$

is nonsingular. So, for all $\tilde{D}, D \in \mathcal{D}_{[0,1]}^{m}$, we have

$$
\begin{aligned}
m & =\operatorname{rank}\left(\left(A^{+} B+D+I\right) \tilde{D}+I-\tilde{D}\right)=\operatorname{rank}\left(A^{+} B \tilde{D}+D \tilde{D}+I\right) \\
& =\operatorname{rank}\left(A^{+} B \tilde{D}+A^{+} A D \tilde{D}+A^{+} A\right)=\operatorname{rank}\left(A^{+}(A D \tilde{D}+B \tilde{D}+A)\right) .
\end{aligned}
$$

Since $\operatorname{rank}\left(A^{+}\right)=m$, the assertion follows from the property of the rank of a product of matrices.

\section{REFERENCES}

[1] T. Ando. Totally positive matrices. Linear Algebra Appl., 90:165-219, 1987.

[2] L Elsner, V. Monov, and T. Szulc. On some properties of convex matrix sets characterized by P-matrices and block P-matrices. Linear Multilinear Algebra, 50:199-218, 2002.

[3] L. Guttman. Enlargement methods for computing the inverse matrix. Ann. Math. Statist., 17:336-343, 1946.

[4] R.A. Horn and C.R. Johnson. Matrix Analysis. Cambridge University Press, Cambridge, 1985.

[5] C.R. Johnson and M.J. Tsatsomeros. Convex sets of nonsingular and P-matrices. Linear Multilinear Algebra, 38:233-239, 1995.

[6] B. Kolodziejczak and T. Szulc. Convex combinations of matrices-full rank characterization. Linear Algebra Appl., 287:215$222,1999$.

[7] M. Marcus and H. Minc. A Survey of Matrix Theory and Matrix Inequalities. Dover Publications, New York, 1964.

[8] G. Marsalia and G.P.H. Styan. Equalities and Inequalities for Ranks of Matrices. Linear Multilinear Algebra, 2:269-296, 1974.

[9] C.B. Soh. Schur stability of convex combination of matrices. Linear Algebra Appl., 128:159-168, 1990.

[10] M.J. Tsatsomeros. Lecture Notes on Matrices with Positive Principal Minors: Theory and Applications. Indian Institute of Technology, Madras, 2017. 